\title{
MuSCULOSKELETAL
}

\section{Magnetic resonance imaging of ankle ligaments: A pictorial essay}

\author{
Yogini Nilkantha Sawant, Darshana Sanghvi \\ Department of Radiology, Kokilaben Dhirubhai Ambani Hospital, Mumbai, Maharashtra, India
}

Correspondence: Dr. Yogini Nilkantha Sawant, Department of Radiology, Kokilaben Dhirubhai Ambani Hospital, Four Bungalows, Andheri (West), Mumbai - 400 053, Maharashtra, India. E-mail: dr.yogini sawant@yahoo.co.in

\begin{abstract}
Ankle trauma is commonly encountered and is most often a sprain injury affecting the ligaments. Accurate diagnosis and appropriate treatment rest on knowledge of complex ligamentous anatomy of ankle and the entire spectrum of pathologies. Magnetic resonance imaging (MRI) is the imaging modality of choice for diagnosing ligament pathologies because of its multiplanar capability and high soft tissue contrast. With MRI, it is possible to triage and attribute the cause of post traumatic ankle pain to bone, ligament, or tendon pathologies, which otherwise overlap clinically. In this pictorial essay, emphasis is given to the intricate and unique anatomy and orientation of ankle ligaments. Pathologies of ankle ligaments have been elaborated.
\end{abstract}

Key words: Ankle ligaments; magnetic resonance imaging; sprain; tear

\section{Introduction}

Ankle is a complex mechanism consisting of two joints: the true ankle joint and the subtalar joint. The true ankle joint is composed of three bones, seen from the front: tibia medially; fibula laterally; and talus inferiorly. The true ankle joint is responsible for dorsiflexion and plantar flexion of the foot. Below the true ankle joint is the subtalar joint, composed of calcaneum inferiorly, and talus superiorly. The subtalar joint is responsible for inversion and eversion of the foot.

The two joints are supported and maintained by ligaments, each connecting two bones and the tendons. Injury to the ankle joint is a common emergency and usually a sprain related to sports, walking, fall, or road traffic accident. In ankle sprain, ligaments are frequently injured in association with bone fractures, avulsion, and contusions.

\begin{tabular}{|l|l|}
\hline \multicolumn{2}{|c|}{ Access this article online } \\
\hline Quick Response Code: & \\
\hline & Website: \\
& www.ijri.org \\
& DOI: \\
\hline
\end{tabular}

\section{Imaging protocol}

The sequences and parameters for optimal high resolution images are described in Table 1.

\section{Anatomy}

There are three major groups of ligaments supporting the ankle joint: lateral, medial or deltoid (superficial and deep component) and syndesmotic.

Sketch diagrams representing these groups are shown in Figures 1 and 2.

\section{Lateral ligaments}

Anterior talofibular ligament (ATFL), Posterior talofibular ligament (PTFL), and Calcaneofibular ligament (CFL) [Figure 3A and B]. Use of oblique coronal plane (CFL view) helps in better evaluation of anatomy and pathology (injury) of CFL as compared to orthogonal plane. ${ }^{[1]}$

This is an open access journal, and articles are distributed under the terms of the Creative Commons Attribution-NonCommercial-ShareAlike 4.0 License, which allows others to remix, tweak, and build upon the work non-commercially, as long as appropriate credit is given and the new creations are licensed under the identical terms.

For reprints contact: reprints@medknow.com

Cite this article as: Sawant $Y N$, Sanghvi D. Magnetic resonance imaging of ankle ligaments: A pictorial essay. Indian J Radiol Imaging 2018;28:419-26. 
Normal thickness of ATFL is $2-3 \mathrm{~mm}$, and CFL is $2 \mathrm{~mm}$.

\section{Medial or deltoid ligaments}

\section{Superficial component of deltoid ligament}

Tibiocalcaneal ligament (TCL), Tibionavicular ligament (TNL), and Tibiospring ligament (TSL) [Figures 4 and 5].

\section{Deep component of deltoid ligament}

Anterior tibiotalar ligament (ATTL) and Posterior tibiotalar ligament (PTTL) [Figure 6].

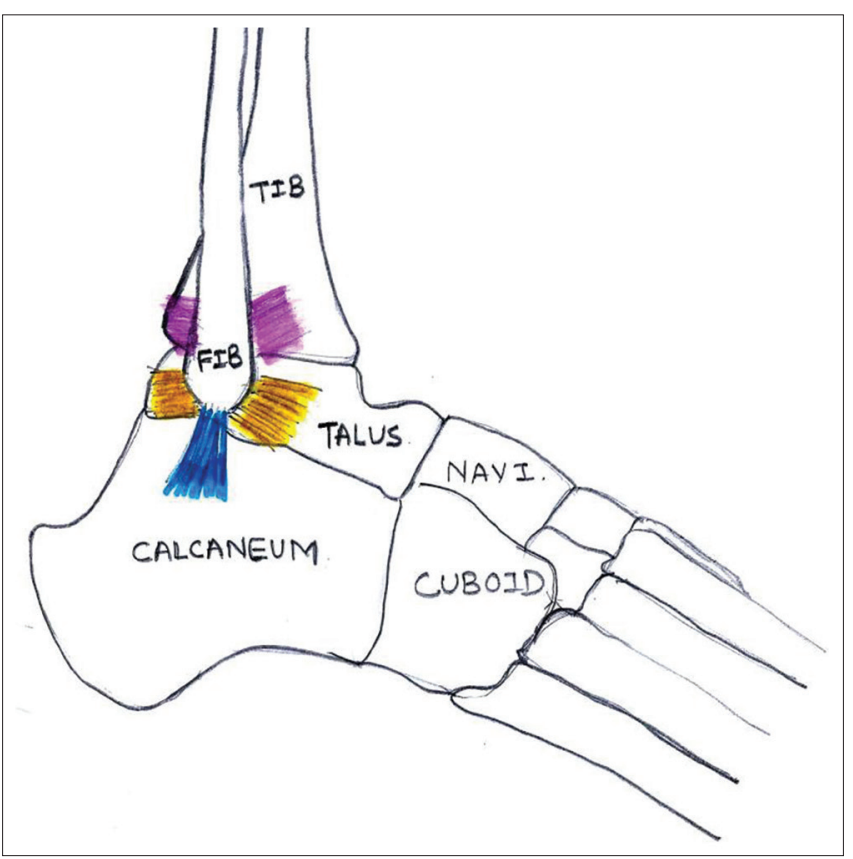

Figure 1: Lateral ligament complex: Anterior and posterior talofibular ligaments (yellow color) and calconeofibular ligament (blue color). Syndesmotic ligaments: Anterior and posterior inferior tibiofibular ligaments (pink color)

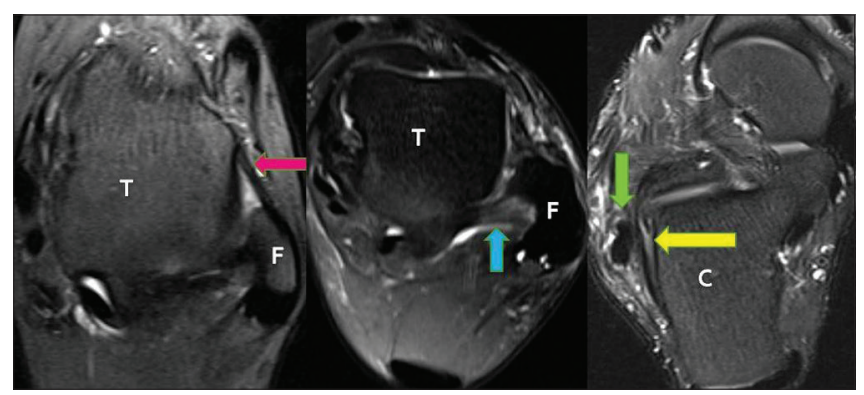

Figure 3 (A): Lateral ligaments: Anterior talofibular ligament and posterior talofibular ligament best identified on axial images, where talar dome $(T)$ is oblong, fibula $(F)$ is ' $\mathrm{C}$ '/crescent shaped. Normal anterior talofibular ligament (pink arrow): Anterolateral tip of lateral malleolus to the neck of talus. Commonly injured. Normal posterior talofibular ligament (blue arrow): Malleolar fossa of lateral malleolus to lateral tubercle of talus. Intracapsular, extrasynovial, strongest, deepest, and least injured of lateral ligaments. Normal calcaneofibular ligament (yellow arrow): Tip of lateral malleolus to trochlear eminence of calcaneum, medial to the peroneal tendons (green arrow), and adjacent to the calcaneum (C)
Normal thickness of TNL is $1-2 \mathrm{~mm}$, and TSL is $2 \mathrm{~mm}$ $(1-4 \mathrm{~mm})$. The PTTL is thickest ligament and normally measures 6-11 $\mathrm{mm}$.

\section{Syndesmotic ligaments}

Anteroinferior tibiofibular ligament (AITFL), Posteroinferior tibiofibular ligament (PITFL), Interosseus membrane or ligament (IOL), and Inferior transverse (ITL). Of these AITFL and PITFL can be easily identified on routine MRI [Figure 7]. Anterior and posterior inferior tibiofibular ligaments are injured simultaneously. ${ }^{[2]}$ A $45^{\circ}$ oblique plane is superior in demonstrating their normal integrity/ pathologies as compared to orthogonal imaging. ${ }^{[3]}$ Fluid in Tibiofibular recess normally measures 5-7 mm.

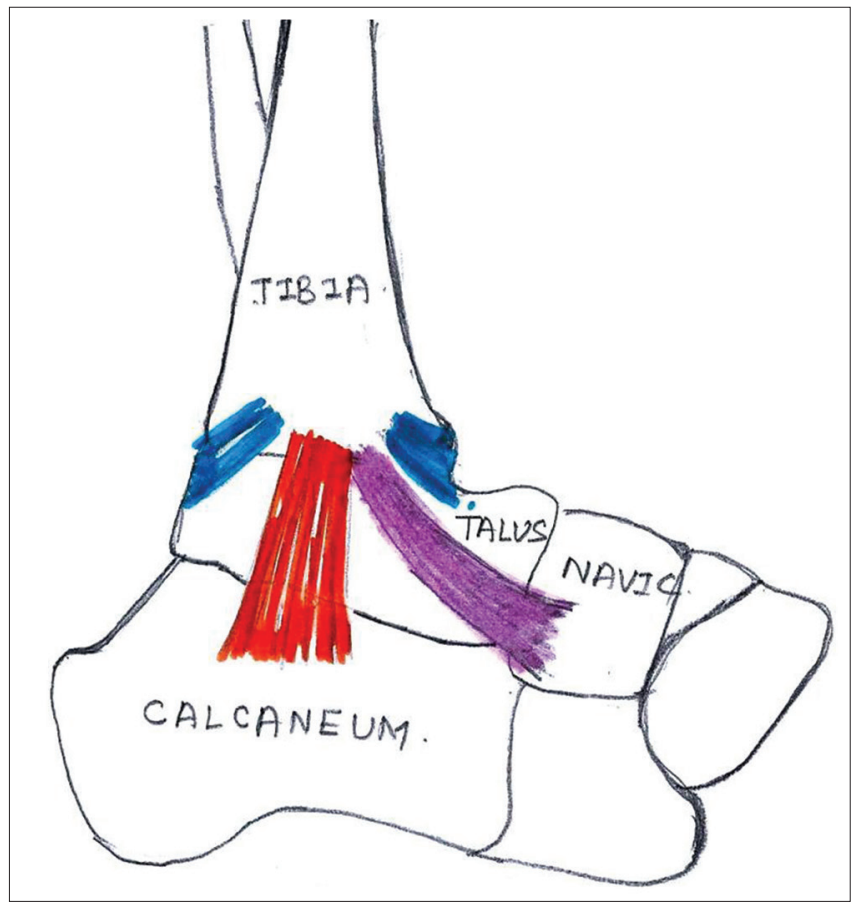

Figure 2: Medial deltoid ligament complex: Superficial deltoid: Tibiocalcaneal ligament (orange color), Tibionavicular ligament (pink color). Deep deltoid: Anterior and posterior tibiotalar ligaments (blue color)

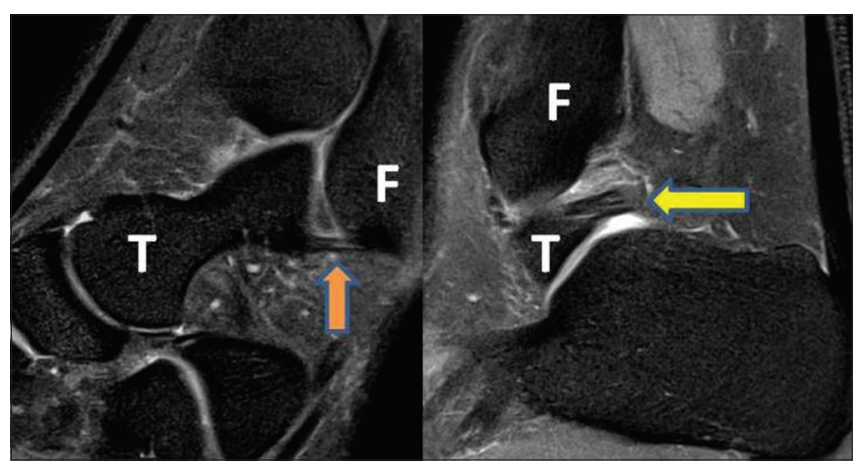

Figure 3 (B): Sagittal proton density fat-saturated image showing anterior talofibular ligament (ATFL): Orange arrow and posterior talofibular ligament (PTFL): Yellow arrow. F: Fibula and T: Talus 
Table 1: Imaging protocol

\begin{tabular}{lccccccc}
\hline Sequences and Parameters & FOV $(\mathrm{mm})$ & TR $(\mathrm{ms})$ & TE $(\mathrm{ms})$ & Mlatrix & Flip angle $\left(^{\circ}\right)$ & Slice thickness $(\mathrm{mm})$ & Inversion $(\mathrm{ms})$ \\
\hline T1 axial/coronal & $120 / 100$ & 600 & 15 & $320 \times 448$ & 140 & 3.0 & 3.0 \\
PD axial/coronal & $120 / 100$ & 3100 & 23 & $275 \times 384$ & 150 & 150 & 3.0 \\
STIR sagittal & $250 / 70$ & 4000 & 29 & $250 \times 384$ & 25 & 0.7 & 210 \\
DESS & $120 / 100$ & 13.01 & 4.79 & $210 \times 256$ & 250
\end{tabular}

FOV: Field of view, TR: Repetition time, TE: Echo time, PD: Proton Density, STIR: Short tau inversion recovery, DESS: Dual echo steady state

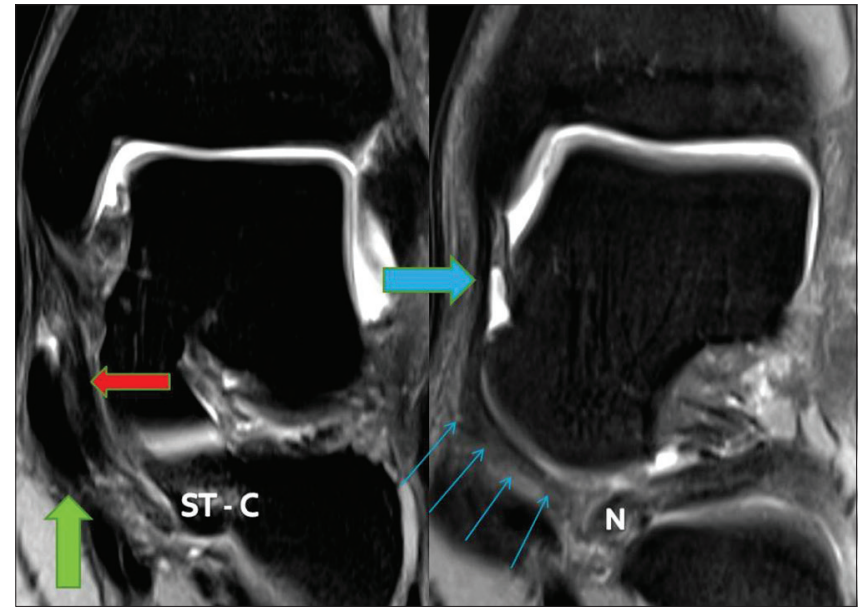

Figure 4: Superficial deltoid ligament (Tibiocalcaneal ligament, Tibionavicular ligament, and Tibiospring ligament): Arise from anterior colliculus of medial malleolus, span two joints, have variable bone attachments distally, and hence, named accordingly. Normal Tibiocalcaneal ligament (red arrow) attaches to the sustentaculum tali of calcaneum (ST- C) and lies medial to tibialis posterior tendon. Tibialis posterior tendon - green arrow. Normal Tibionavicular ligament (blue arrow) attaches to the medial aspect of tuberosity of navicular bone $(\mathrm{N})$, it crosses ankle and talonavicular joints

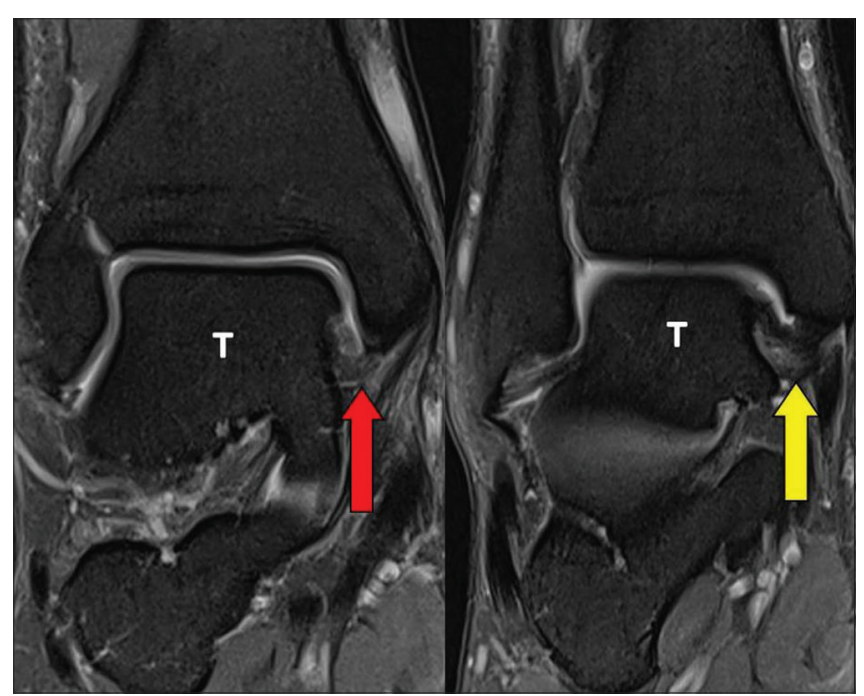

Figure 6: Deep deltoid ligament: arise from intercollicular groove of medial malleolus of tibia, cross only one joint, intra articular and surrounded by synovium. Normal anterior tibiotalar ligament (ATTL: red arrow) is thin, striated, and attaches to the anterior aspect of body of neck of talus bone (T). Normal posterior tibiotalar ligament (PTTL: yellow arrow) is thick, striated, and attaches to the posterior most aspect of medial tubercle of talus bone $(\mathrm{T})$

In addition to these, the ankle is supported by the spring ligament. The spring ligament complex consists

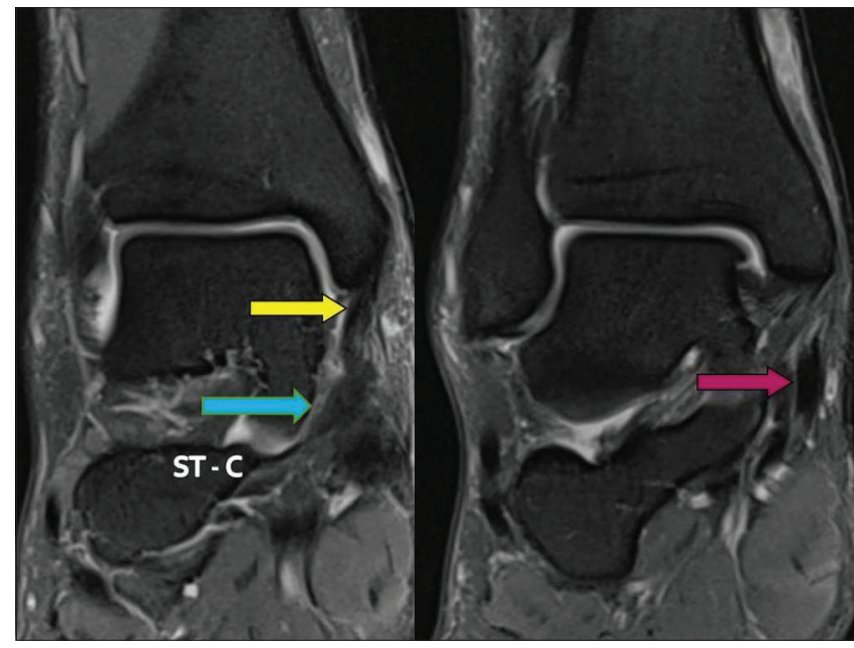

Figure 5: Normal Tibiospring ligament (yellow arrow) is the only component of superficial deltoid ligament with no distal bone attachment, it merges with the superomedial (SM) component of spring ligament (blue arrow). The superomedial component of spring ligament is attached to the sustentaculum tali of calcaneum (ST- C). Tibiospring ligament lies medial to the Tibialis posterior tendon (pink arrow). The Tibiospring ligament, superomedial component of spring ligament complex and Tibialis posterior tendon have synergistic action in maintaining the medial plantar arch

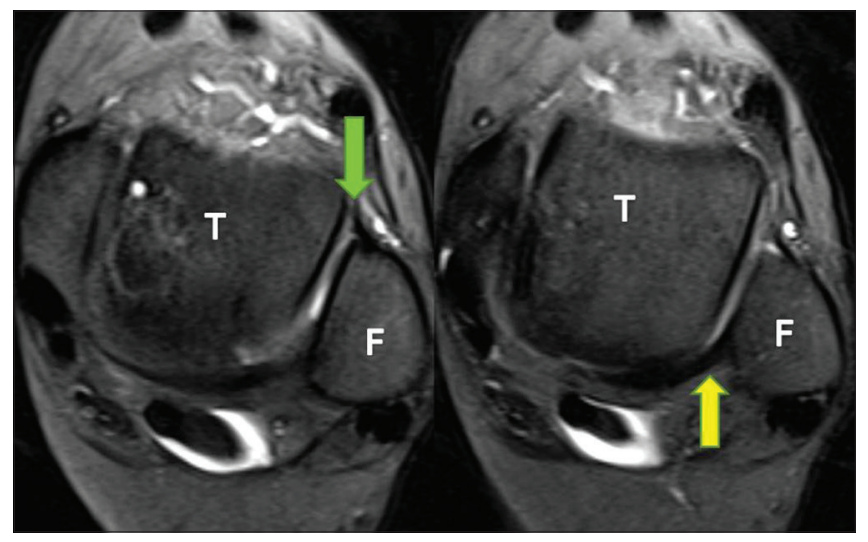

Figure 7: Normal anterior (green arrow) and posterior (yellow arrow) inferior tibiofibular or syndesmotic ligaments are best identified on axial images, where the talar dome $(T)$ is square shaped, and Fibula is round

of superomedial (SM), medioplantar oblique (MPO), and inferoplantar longitudinal (IPL) components [Figures 8 and 9]. Normal thickness of SM component is $3 \mathrm{~mm}(2-5 \mathrm{~mm})$, and IPL component is $4 \mathrm{~mm}(2-6 \mathrm{~mm})$.

\section{Pathology}

Ligaments connect two bones; have higher proteoglycan and water content and less collagen. Normal ligaments 


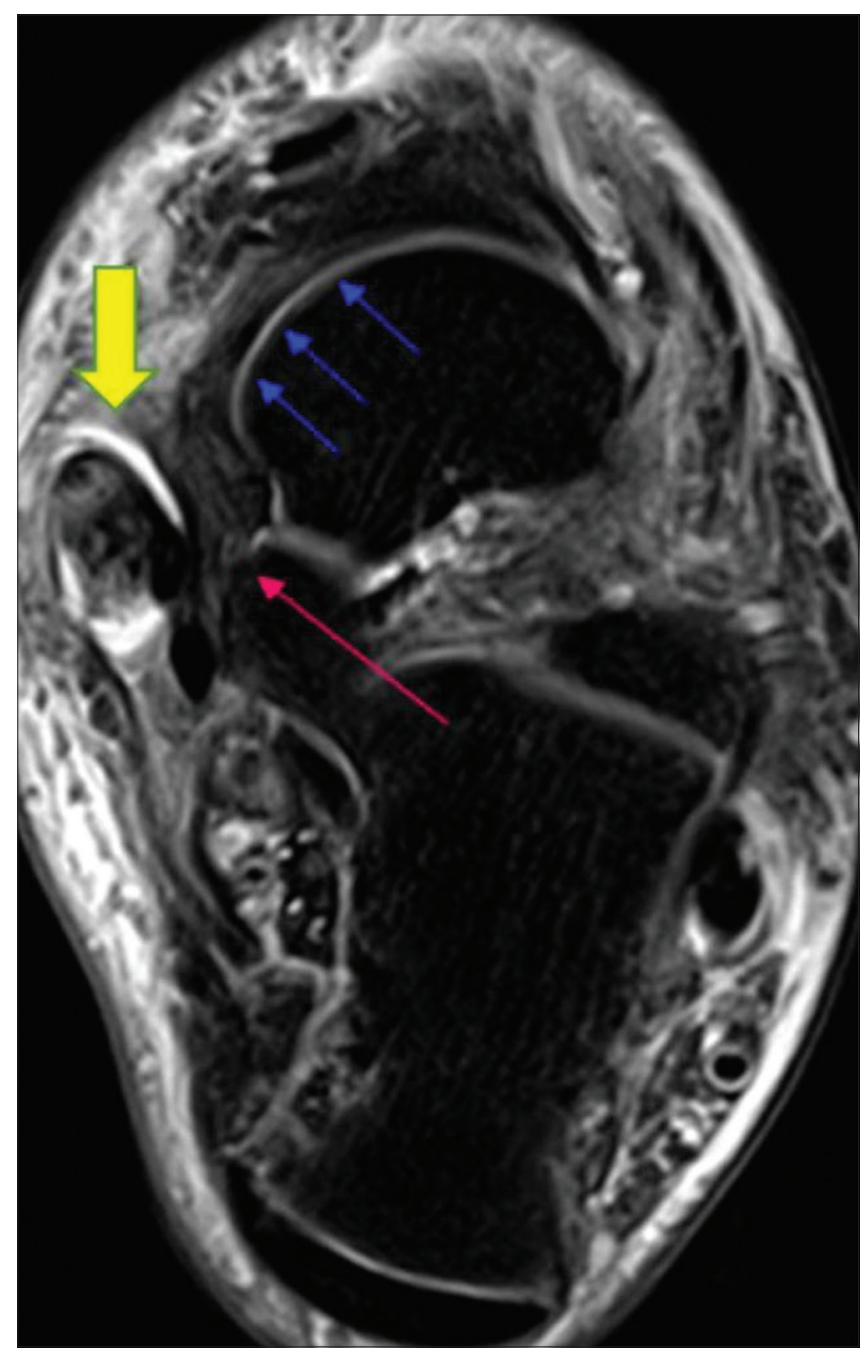

Figure 8: Spring ligament complex includes the Superomedial, Medioplantar, and Inferoplantar longitudinal components. Normal superomedial component of the spring ligament: From sustentaculum tali (pink arrow) to superior and posteromedial aspect of navicular bone (blue arrows). In association with tibiospring ligament, it primarily stabilizes talocalcaneonavicular joint and secondarily stabilizes medial plantar arch of foot. Tibialis posterior tendon tendinosis (yellow arrow)

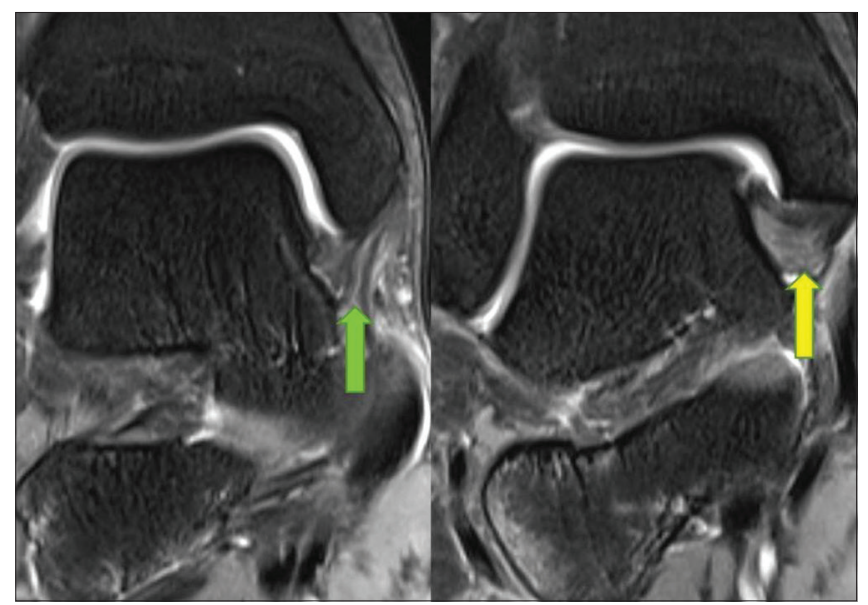

Figure 10: Interstitial tear of anterior tibiotalar ligament (green arrow) and posterior tibiotalar ligament (yellow arrow): Hyperintense signal seen within these ligaments due to the presence of edema or hemorrhage

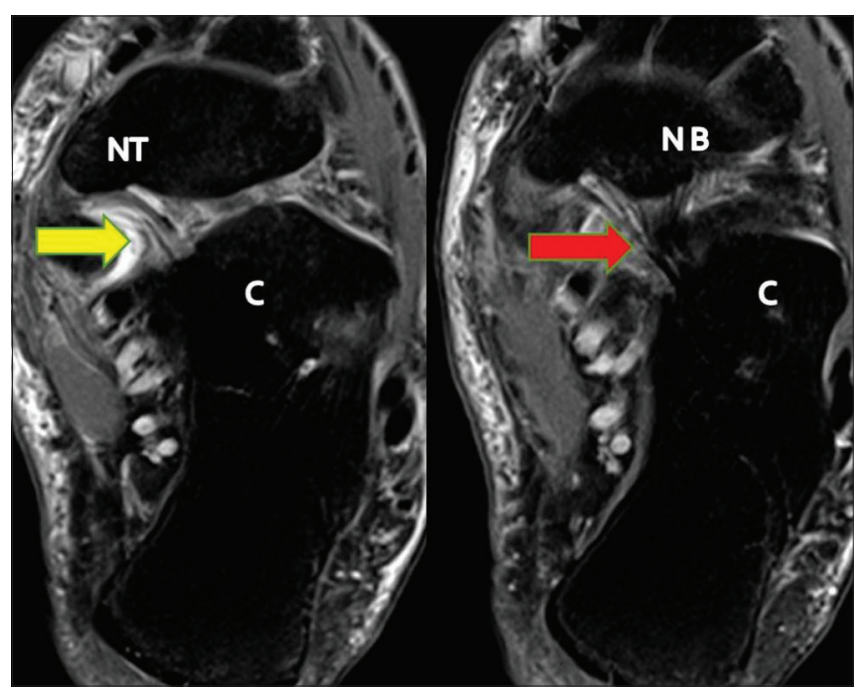

Figure 9: Normal medioplantar oblique component of the spring ligament (yellow arrow): From coronoid fossa of calcaneum (C) to tuberosity of navicular bone. Normal inferoplantar longitudinal component of the spring ligament (red arrow): From coronoid fossa of calcaneum $(\mathrm{C})$ to beak of navicular bone

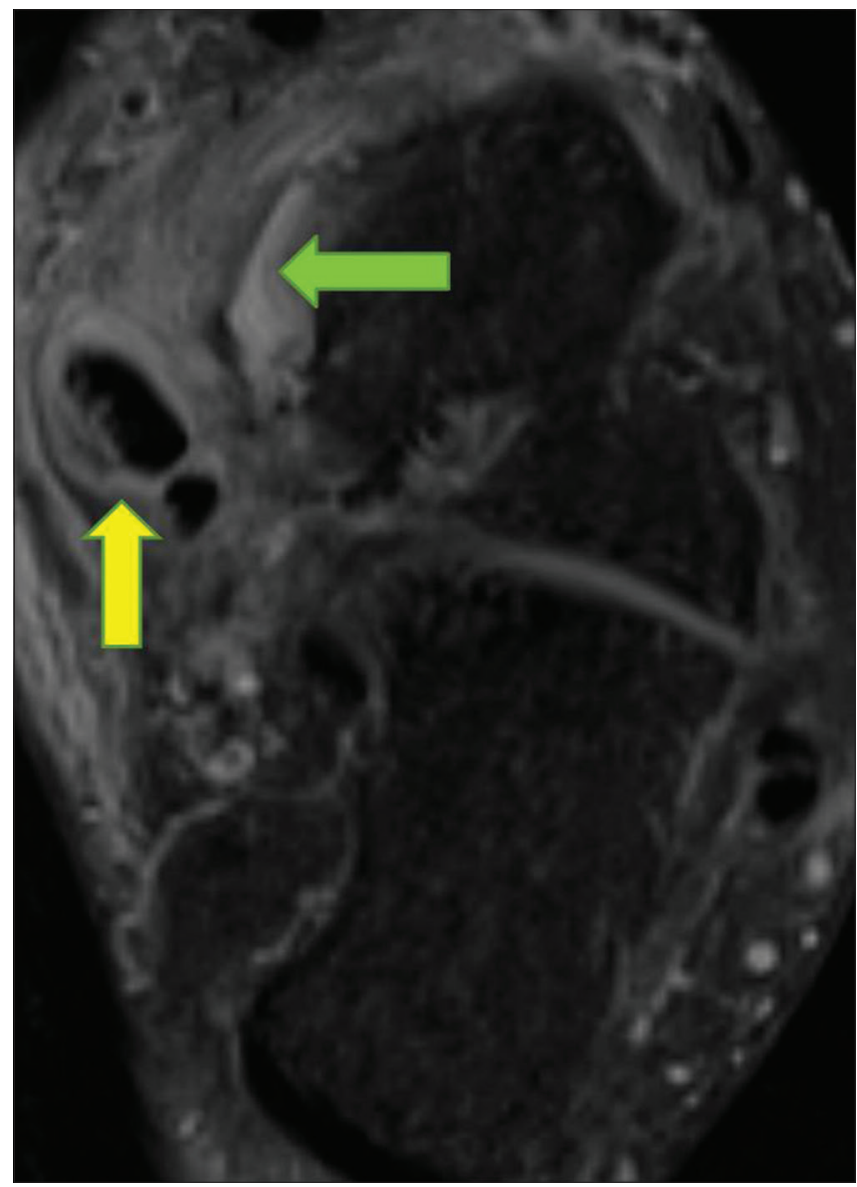

Figure 11: Partial tear of superomedial component of the spring ligament (green arrow): Seen as partial discontinuity, not involving the entire thickness of ligament. Associated tibialis posterior tendinosis (yellow arrow)

are seen as uniform low-intensity band on T1 and T2 W images. ${ }^{[4]}$ Notable exceptions include posterior talofibular, 
deep components of the deltoid ligament, and anterior inferior tibiofibular ligament that show heterogeneous signal and striations because of the interspersed fat between their fascicles.

Following acute injury the ligament undergoes repair in three stages. The first three days are characterized by inflammation, interstitial edema, and hemorrhage. The third to fifth days represent stage of repair with proliferation of fibroblasts. The second to fourth weeks are the stage of remodeling with formation of collagen. Periarticular edema is seen up to the seventh week.

\section{Acute Injury}

MRI shows blurred margins, irregular contours, discontinuous/wavy/lax fibers, inhomogeneous signal, or loss of normal hypointense signal within the ligament. Certain ligaments such as PTFL, deep components of the deltoid ligament, and AITFL show loss of normal striations due to presence of edema and hemorrhage. The ligament may be attenuated, thick, thin, or elongated.

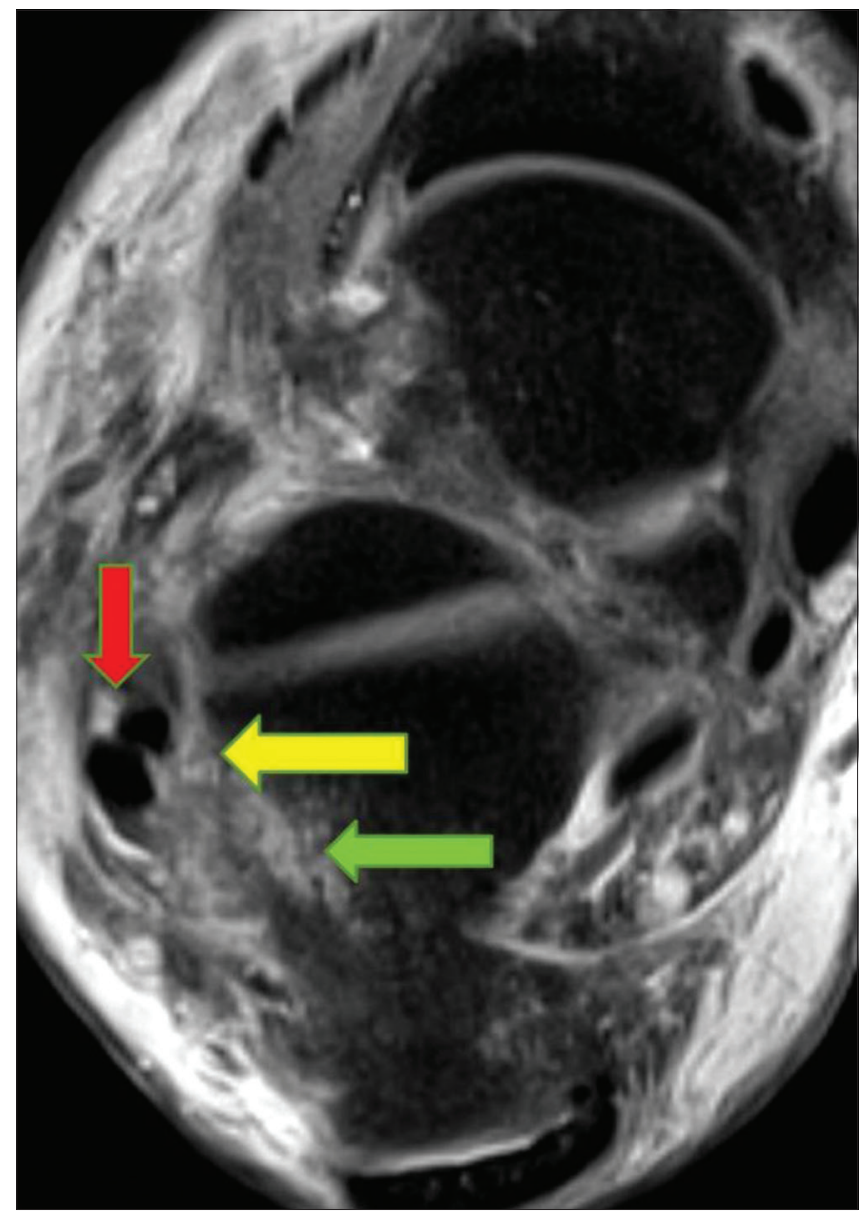

Figure 12: Complete tear of Calcaneofibular ligament (yellow arrow): Seen as discontinuity involving the entire thickness of ligament. Associated peroneal tendinosis (red arrow) and contusions of calcaneum (green arrow)
Acute ligament injuries can be graded as interstitial, partial, or complete tear.

Interstitial tears

Hyperintense signal within the ligament on PD and T2W images due to presence of edema or hemorrhage [Figure 10].

\section{Partial tears}

Partial discontinuity of ligaments reaching up to the surface but not involving the entire thickness of ligament [Figure 11].

\section{Complete tears}

Discontinuity involving the entire thickness of ligament and is seen as fluid filled defect and retraction [Figures 12 and 13]. Joint effusion and obliteration of adjacent fat planes can be seen.

Ankle sprain contributes to $20-40 \%$ of sport injuries. ${ }^{[5]}$ Depending on the mechanism of injuries, particular group of ligaments are injured.

Inversion injury accounts for $85 \%$ of ankle sprain and is commonly seen with high velocity sports such as basketball, soccer, and football. ${ }^{[5,6]}$ Inversion injury commonly affects the lateral ligament complex. Of these, ATFL being the

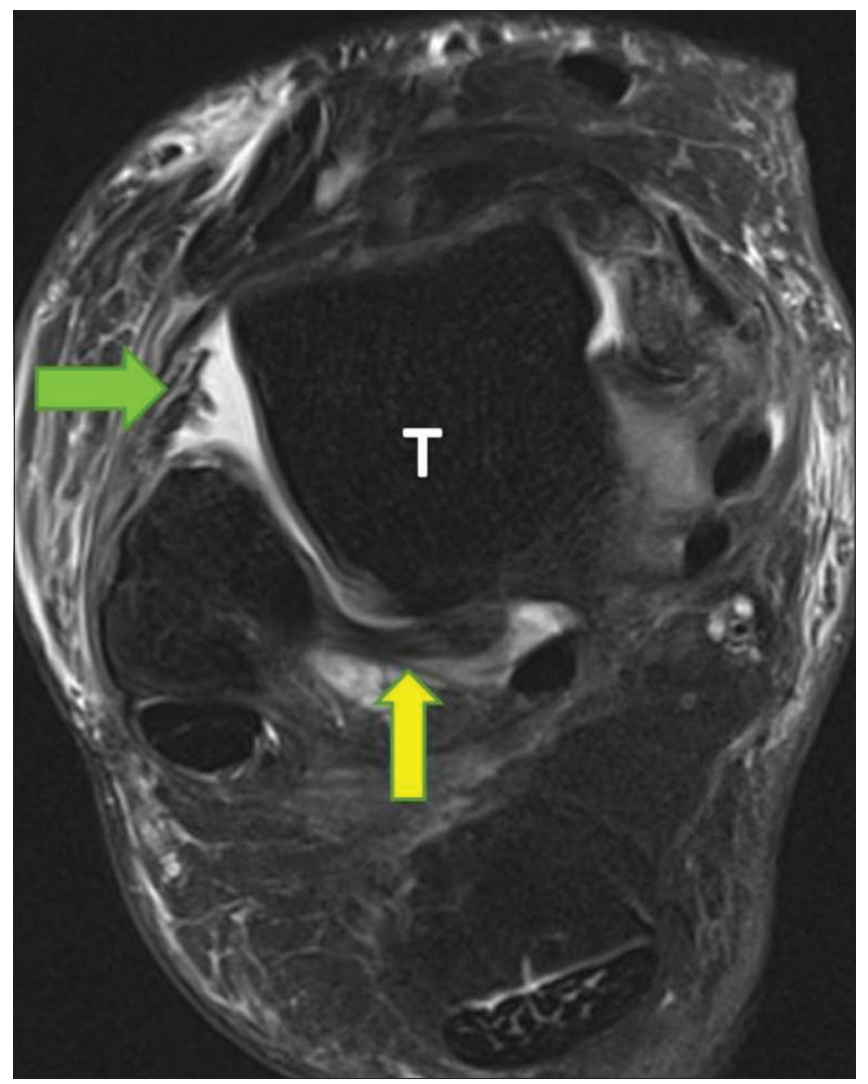

Figure 13: Complete tear of anterior talofibular ligament (green arrow): Seen as discontinuity with retraction of the ligament. Associated fluid seen in the anterolateral gutter. Posterior talofibular ligament is intact (yellow arrow). Talus bone $(\mathrm{T})$ 


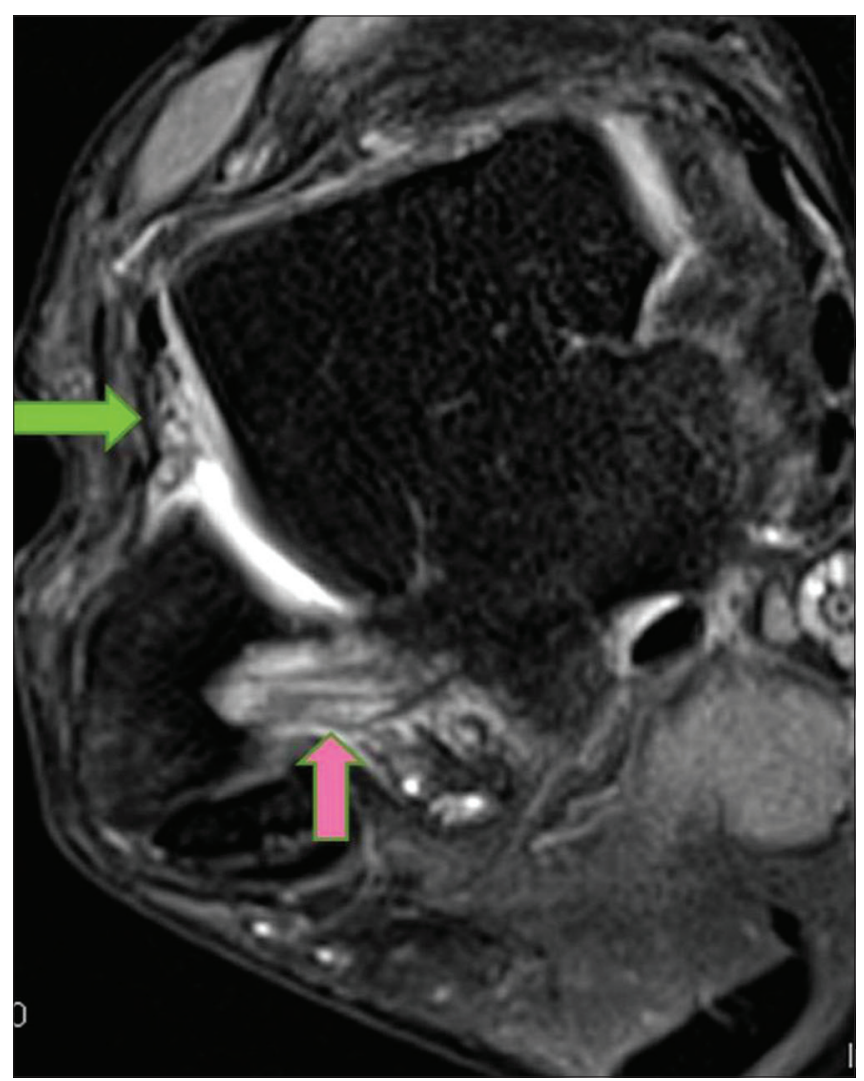

Figure 14: Inversion injury: Complete tear of anterior talofibular ligament (green arrow): Seen as discontinuous ligament. Also seen is partial tear of Posterior talofibular ligament (pink arrow) with edema and loss of striations. Associated fluid in the anterolateral gutter

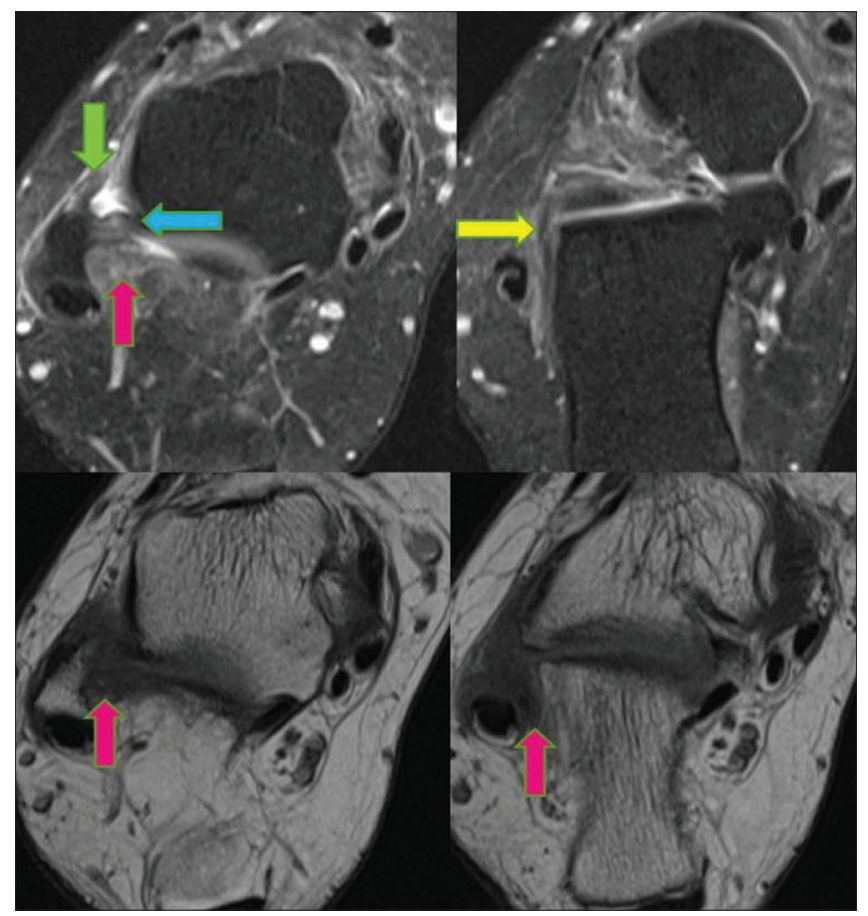

Figure 16: Anterolateral impingement syndrome: Chronic partially healed tears of anterior talofibular ligament (green arrow), posterior talofibular ligament (blue arrow), and calcaneofibular ligament (yellow arrow). Soft tissue in the anterolateral gutter (pink arrows) with synovial hyperplasia represents 'meniscus'

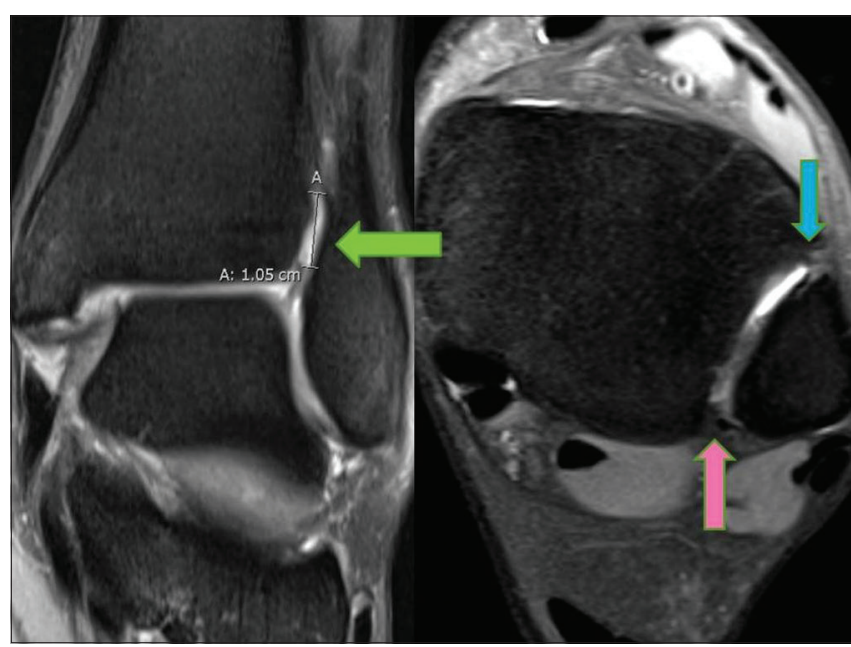

Figure 15: High ankle sprain: Fluid in the tibiofibular recess measures approximately, $10 \mathrm{~mm}$ (green arrow) with torn anterior and posterior inferior tibiofibular/syndesmotic ligaments (blue and pink arrows, respectively). Normal tibiofibular recess measures 5-7 mm, approximately

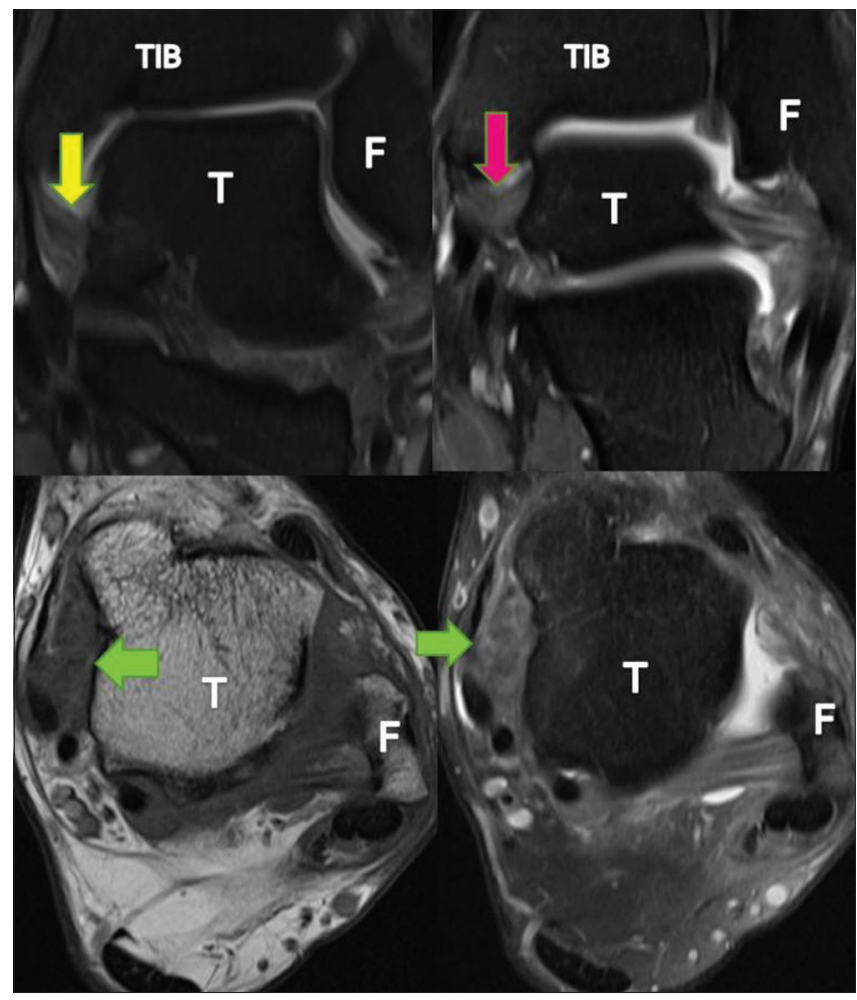

Figure 17: Medial impingement syndrome: Chronic partially healed tears of the anterior tibiotalar ligament (yellow arrow) and posterior tibiotalar ligament (pink arrow). Soft tissue in the anteromedial gutter (green arrows) with hypertrophic synovium represents 'meniscus'. TIB: Tibia, F: Fibula, T: Talus

weakest is more prone to injury ${ }^{[7,8]}$ [Figure 14]. Injury to ATFL is less common at the talar attachment because of the denser fibrocartilage and greater bone density than that of the fibular attachment. ${ }^{[9]}$

Eversion injuries commonly affect the medial deltoid ligament complex, accounting for approximately 5\% 


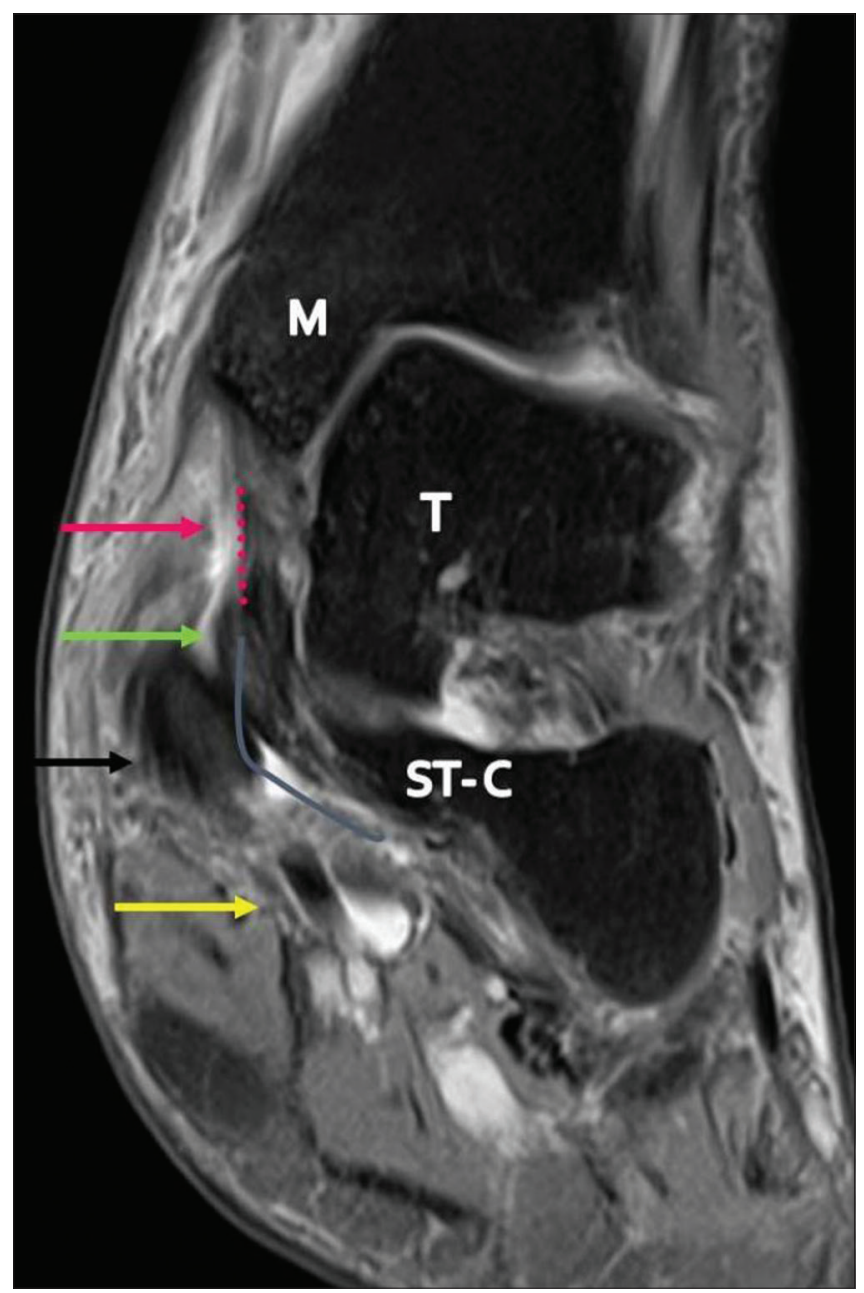

Figure 18: Synergistic action of tibialis posterior tendon, tibiospring ligament, and superomedial component of the spring ligament complex in maintaining the medial plantar arch. Partial tear of tibiospring ligament (pink dotted line and arrow), sprain of superomedial component of spring ligament (gray curved line and green arrow). Tibialis posterior tendinosis and partial interstitial tear (black arrow). Normal flexor digitorum longus tendon (yellow arrow). M: Medial malleolus of Tibia, ST-C: Sustentaculum tali of calcaneum, T: Talus

of ankle sprains and are associated with sports such as gymnastic, rugby, and soccer. ${ }^{[10,11]}$

High ankle/syndesmotic sprains account for $7 \%$ of routine ankle sprains and $40 \%$ of injuries in athletes. These are more often seen in association with sports such as football, ice hockey, soccer, skiing, running, and jumping. ${ }^{[12,13]}$ Fluid in the tibiofibular recess measuring $>12 \mathrm{~mm}$ is definitely abnormal and suggestive of acute injury to syndesmotic ligaments or high ankle sprains $^{[14,15]}$ [Figure 15].

\section{Chronic Injury}

MRI shows an attenuated, thin - hypoplastic, or alternately a thick - hyperplastic ligament with irregular contours and absence of edema or hemorrhage.

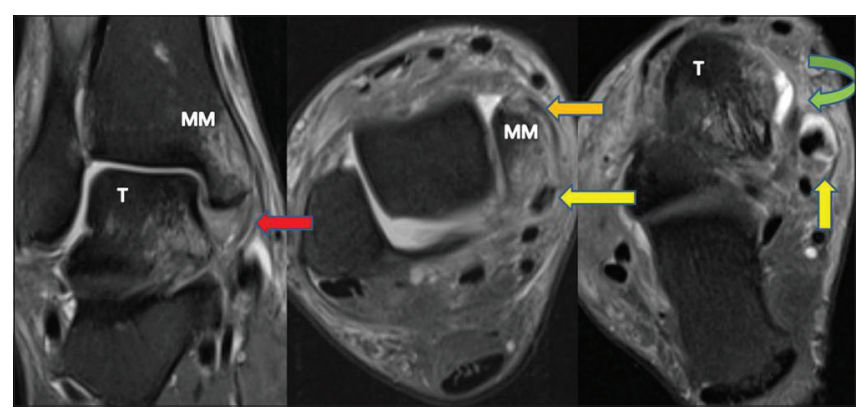

Figure 19: Bone contusions in medial malleolus (MM), joint effusion, and Tibialis posterior tendinosis (yellow arrows) associated with tear involving the deep Deltoid - posterior tibiotalar ligament (red arrow) and superficial Deltoid - tibiocalcaneal ligament (orange arrow) and tibiospring ligament (green arrow). T: Talus

\section{Impingement Syndrome}

Anatomical variations of the ligaments alter joint mechanics, thus, making the individual prone to repeated injuries and chronic instability ${ }^{[16]}$ Anterolateral and medial impingement syndromes are the result of such chronic repetitive stress; these can be adequately diagnosed on MRI.

In chronic ATFL tear, granulation/scar tissue or fibrosis in the anterolateral gutter resembles a "meniscus" and can lead to clinical anterolateral impingement syndrome [Figure 16]. Similar findings can be seen in anteromedial and posteromedial impingement syndromes [Figure 17].

Post contrast fat-suppressed 3D-FSPGR (3D fast spoiled gradient echo recalled acquisition in the steady state) sequence of the ankle is superior and accurate in assessing anterolateral impingement syndrome by better delineation of synovial thickening/inflammation and granulation tissue. ${ }^{[8]}$

\section{Important Associations}

Ligaments and adjacent tendons have synergistic functions, and their injuries are most often simultaneous. Peroneal tendinosis is commonly seen with ATFL injury followed by CFL and PTFL injury ${ }^{[17]}$ [Figure 12]. Partial longitudinal tear of peroneus brevis tendon is commonly associated with lateral ankle sprain. ${ }^{[18]}$ Tibialis posterior tendon injuries are seen simultaneously with tibiospring and spring ligament complex injuries because of their synergistic action in maintaining the medial plantar $\operatorname{arch}^{[19,20]}$ [Figures 18 and 19]. Bone contusion, osteochondral lesion, and joint effusion are other common associations [Figure 19].

\section{Conclusion}

Knowledge of anatomy and imaging appearance of normal and abnormal ankle ligaments on MRI with an understanding of biomechanics of injury aids in achieving accurate diagnosis and appropriate treatment of ankle 
sprains. Depending on the MRI features and the clinical co-relates (Drawers test, inversion and eversion tests), it is possible for the clinicians to grade the ankle injury from mild to severe and also assess instability. Most of the ankle sprains are stable and are treated conservatively (rest, immobilization, ice compression, and elevation). Those associated with osteochondral lesions are treated with arthroscopic repair. Severe ankle sprain injuries with instability are treated with surgical reconstruction of ligaments.

\section{Financial support and sponsorship}

Nil.

\section{Conflicts of interest}

There are no conflicts of interest.

\section{References}

1. Park HJ, Lee SY, Park NH, Kim E, Chung EC, Kook SH, et al. Usefulness of the oblique coronal plane in ankle MRI of the calcaneofibular ligament. Clin Radiol 2015;70:416-23.

2. Dattani R, Patnaik S, Kantak A, Srikanth B, Selvan TP. Injuries to the tibiofibular syndesmosis. J Bone Joint Surg Br 2008;90:405-10.

3. Hermans JJ, Ginai AZ, Wentink N, Hop WC, Beumer A. The additional value of an oblique image plane for MRI of the anterior and posterior distal tibiofibular syndesmosis. Skeletal Radiol 2011;40:75-83.

4. Hodgson RJ, O'Connor PJ, Grainger AJ. Tendon and ligament imaging. Br J Radiol 2012;85:1157-72.

5. Dubin JC, Comeau D, McClelland RI, Dubin RA, Ferrel E. Lateral and syndesmotic ankle sprain injuries: A narrative literature review. J Chiropr Med 2011;10:204-19.

6. Purcell SB, Schuckman BE, Docherty CL, Schrader J, Poppy W. Differences in ankle range of motion before and after exercise in 2 tape conditions. Am J Sports Med 2009;37:383-9.

7. Boruta PM, Bishop JO, Braly WG, Tullos HS. Acute lateral ankle ligament injuries: A literature review. Foot Ankle 1990; 11:107-13.

8. Choo HJ, Suh JS, Kim SJ, Huh YM, Kim MI, Lee JW, et al. Ankle MRI for anterolateral soft tissue impingement: Increased accuracy with the use of contrast-enhanced fat-suppressed 3D-FSPGR MRI. Korean J Radiol 2008; 9:409-15.

9. Kumai T, Takakura Y, Rufai A, Milz S, Benjamin M. The functional anatomy of the human anterior talofibular ligament in relation to ankle sprains. J Anat 2002; 200:457-65.

10. Hintermann B, Knupp M, Pagenstert GI. Deltoid ligament injuries: Diagnosis and management. Foot Ankle Clin 2006; 11:625-37.

11. Waterman BR, Belmont PJ Jr., Cameron KL, Svoboda SJ, Alitz CJ, Owens BD, et al. Risk factors for syndesmotic and medial ankle sprain: Role of sex, sport, and level of competition. Am J Sports Med 2011; 39:992-8.

12. Boytim MJ, Fischer DA, Neumann L. Syndesmotic ankle sprains. Am J Sports Med 1991; 19:294-8.

13. Norkus SA, Floyd RT. The anatomy and mechanisms of syndesmotic ankle sprains. J Athl Train 2001; 36:68-73.

14. Brown KW, Morrison WB, Schweitzer ME, Parellada JA, Nothnagel $\mathrm{H}$. MRI findings associated with distal tibiofibular syndesmosis injury. AJR Am J Roentgenol 2004; 182:131-6.

15. Takao M, Ochi M, Naito K, Iwata A, Kawasaki K, Tobita M, et al. Arthroscopic diagnosis of tibiofibular syndesmosis disruption. Arthroscopy 2001; 17:836-43.

16. Imhauser CW, Siegler S, Udupa JK, Toy JR. Subject-specific models of the hindfoot reveal a relationship between morphology and passive mechanical properties. J Biomech 2008; 41:1341-9.

17. Ziai P, Benca E, Wenzel F, Schuh R, Krall C, Auffahrt A, et al. Peroneal tendinosis as a predisposing factor for the acute lateral ankle sprain in runners. Knee Surg Sports Traumatol Arthrosc 2016; 24:1175-9.

18. Karlsson J, Wiger P. Longitudinal split of the peroneus brevis tendon and lateral ankle instability: Treatment of concomitant lesions. J Athl Train 2002; 37:463-6.

19. Hintermann B. Biomechanics of the unstable ankle joint and clinical implications. Med Sci Sports Exerc 1999; 31:S459-69.

20. Deland JT, de Asla RJ, Sung IH, Ernberg LA, Potter HG. Posterior tibial tendon insufficiency: Which ligaments are involved? Foot Ankle Int 2005; 26:427-35. 